\title{
The case for no field decay from improved pulsar population studies
}

\author{
J.W. Hartman, F. Verbunt \\ Astronomical Institute, Utrecht, the Netherlands \\ D. Bhattacharya
}

Raman Research Institute, Bangalore, India

R. Wijers

Institute of Astronomy, Cambridge, UK

\section{Introduction}

We perform radio pulsar population synthesis to study the evolution of the pulsar population. In doing this, we continue our earlier work on this subject (Bhattacharya et al. 1992). We have extended our work by 1) calculating orbits in the whole galaxy (the simulation of the observations however is still limited to the solar neighborhood), 2) using the high birth velocities of radio pulsars from Lyne \& Lorimer (1994) and 3) implementing the new model by Taylor \& Cordes (1993) of the distribution of free electrons in the galaxy. A full report of this work will be given in Hartman et al. (1996).

\section{Simulations}

We have generated simulated samples of pulsars for several combinations of input parameters, i.e. the initial magnetic field strength distribution, the luminosity distribution, the decay time of the magnetic field strength and the initial velocity distribution. We find that models with long $(100 \mathrm{Myr})$ decay times describe the observations much better than models with shorter decay times $(10 \mathrm{Myr})$. In the latter case, for a simulation with a well fitted magnetic field strength distribution, the simulated populations contain too few long period pulsars compared to the observed numbers. An initial magnetic field strength with on average higher magnetic fields will produce a correct period distribution, but also a magnetic field distribution with too many high magnetic field pulsars. Another approach is favoring these long period pulsars in the luminosity distribution, which is dependent on the period and period derivative. However, the adjustment in the luminosity distribution needed to get a sufficient number of long period pulsars results in too many high luminosity pulsars at large distances. This problem does not occur for a decay time of $100 \mathrm{Myr}$, since we can fit both the period and the magnetic field strength distribution. A change in the luminosity distribution with respect to our earlier calculations is required due to the increased distance scale of nearby pulsars, as a result of the Taylor \& Cordes electron density 

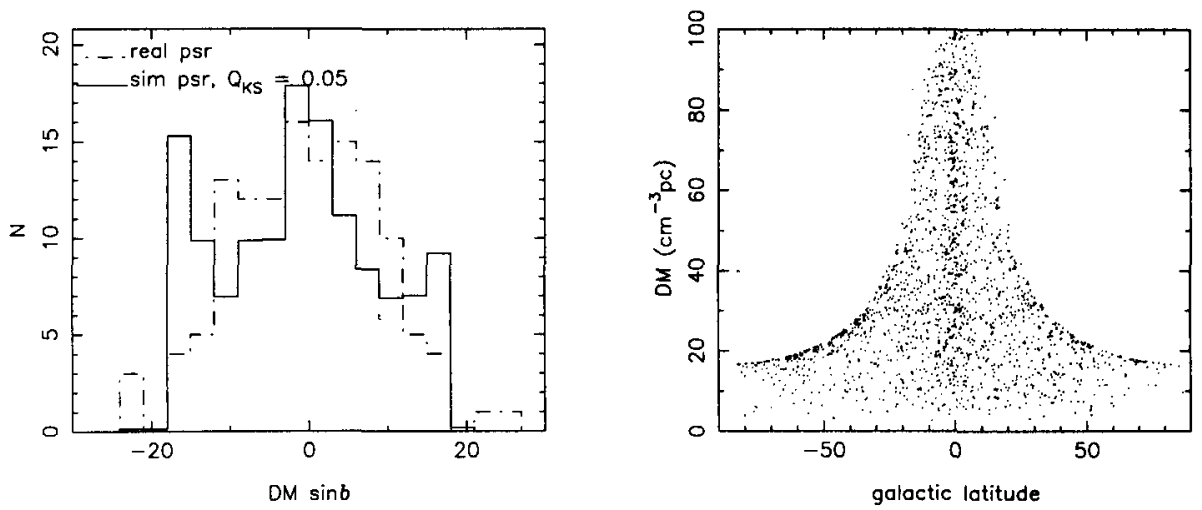

Figure 1. The left panel shows a histogram of the dispersion measure perpendicular to the galactic plane of 2000 pulsars, simulated using a decay time of $100 \mathrm{Myr}$, normalized to 129 pulsars. The dash-dotted line represents the observed sample of 129 pulsars. Indicated in the diagram is the Kolmogorov-Smirnov probability that the two distributions are drawn from the same mother distribution. The right panel shows the simulated distribution of the dispersion measure as a function of galactic latitude. The maximum dispersion measure from the Taylor \& Cordes model is clearly visible.

distribution model. We do have problems fitting the dispersion measure, since the Taylor \& Cordes model features a sharp edge in the maximum dispersion measure that is not present in the observed sample, as is shown in figure 1.

We also find that simulations that use an initial velocity distribution with many more pulsars at low velocity (Phinney's modification of the Paczyński distribution, see Pazcyński 1990) describe the period and magnetic field distribution equally well as simulations that use the Lyne \& Lorimer velocity distribution.

\section{References}

Bhattacharya, D., Wijers, R., Hartman, J., Verbunt, F. 1992, A\&A, 254, 198 Hartman, J., Verbunt, F., Bhattacharya, D., Wijers, R. 1996, A\&A in prep. Lyne, A., Lorimer, D. 1994, Nat, 369, 127

Paczyński, B. 1990, ApJ, 348, 485

Taylor, J., Cordes, J. 1993, ApJ, 411, 674 